\title{
Generalized Fierz identities and applications to spin-3/2 particles
}

\author{
Yi Liaod1, Ji-Yuan Liu ${ }^{b}$ \\ ${ }^{a}$ School of Physics, Nankai University, Tianjin 300071, China \\ ${ }^{b}$ College of Science, Tianjin University of Technology, Tianjin 300384, China
}

\begin{abstract}
We study the most general Fierz identities for a pair of non-contracted Dirac matrices both in the standard basis and for chiral spinors. These identities are useful in building independent effective operators of fermions that involve derivatives or sextuple and more fields. We apply them to construct independent effective four-fermion interactions that contain one to three spin-3/2 chiral fields. These operators could be relevant to physics of higher-spin baryons and phenomenology of a neutral, massive spin-3/2 particle as a dark matter candidate.
\end{abstract}

\footnotetext{
${ }^{1}$ liaoy@nankai.edu.cn
} 


\section{Introduction}

The Fierz identities are a set of relations that reshuffle a pair of fermion fields in a product of bilinear forms. The identities were originally built for a scalar product of two bilinear forms made of spin-1/2 fields, relating $\bar{\psi}_{1} \Gamma^{A} \psi_{2} \bar{\psi}_{3} \Gamma_{A} \psi_{4}$ to a sum of the terms $\bar{\psi}_{1} \Gamma^{B} \psi_{4} \bar{\psi}_{3} \Gamma_{B} \psi_{2}$ [1, 2]. Here $\Gamma^{A}$ refers to a set of sixteen matrices that are classified as the scalar, pseudoscalar, vector, axial-vector and tensor, and that together form the standard basis for $4 \times 4$ matrices. This was generalized some years ago [3] to the case of the pseudoscalar products, $\bar{\psi}_{1} \Gamma^{A} \psi_{2} \bar{\psi}_{3} \tilde{\Gamma}_{A} \psi_{4}$, where $\tilde{\Gamma}^{A}$ stands for the parity-opposite counterpart of $\Gamma^{A}$, and the case in which one reshuffles with the help of charge conjugation a barred and an unbarred spinor (e.g., $\psi_{2}$ and $\bar{\psi}_{3}$ ) in a scalar or pseudoscalar product.

In practical work we sometimes come across a product $\bar{\psi}_{1} \Gamma^{A} \psi_{2} \bar{\psi}_{3} \Gamma_{B} \psi_{4}$ where the two indices are not or only partially contracted. Reshuffling a pair of spinors in this case requires further generalized Fierz identities for a non-contracted product. Such an identity was first explicitly written down in Ref. [4] for the product, $\bar{\psi}_{1} \sigma_{\mu \nu} P_{-} \psi_{2} \bar{\psi}_{3} \gamma^{v} P_{+} \psi_{4}$, where $P_{ \pm}=\left(1 \pm \gamma_{5}\right) / 2$ are the projectors for the right- and left-handed spinors. It proves to be very useful to remove the redundancy [5] in the leading, dimension-seven, parity-violating four-nucleon operators in which one Lorentz index is carried by a derivative [6]. A second circumstance in which such generalized identities are necessary involves reshuffling spinors in an operator containing six or more spinor fields. An outstanding example of this is the instanton-induced 't Hooft six-quark interaction [7] that respects the chiral $S U(3)$ but breaks the axial $U(1)$ symmetries. Generalized identities are useful to recast the interaction in a form that would meet the special needs in the mean-field approach to the color-superconductor [8]. Finally, non-contracted pairs of gamma matrices appear definitely in less studied four-fermion operators where spin-3/2 (or higher) fields are present which carry a Lorentz index. These operators are relevant to hadron physics that describes effective interactions between the spin-3/2 and spin-1/2 baryons. Rather recently a neutral spin-3/2 particle has been suggested as a dark matter candidate and its four-fermion effective interactions were phenomenologically studied [9, 10, 11].

In this work we conduct a systematic analysis on the non-contracted Fierz identities. We first do so in the next section in the standard basis of the gamma matrices. This is followed by section 3 in which chiral spinors are employed. The identities turn out to be much simpler than in the standard basis, and their usefulness is demonstrated by a few examples of sextuple forms of spinors. In section 4 we construct the quadruple forms involving one to three vector-spinors corresponding to spin-3/2 particles, and employ the generalized Fierz identities established in this work to examine their independence. We briefly summarize in the last section.

\section{Identities in the standard basis}

The Fierz identities are based on the completeness of a set of 16 matrices for the $4 \times 4$ matrices. For the standard basis we choose the following one:

$$
\Gamma^{A}=1 ; \gamma_{5} ; \gamma^{a} ; \gamma^{a} \gamma_{5} ; \sigma^{a_{1} a_{2}}\left(a_{1}<a_{2}\right)
$$


We work in the convention: $g^{\mu v}=\operatorname{diag}(+1,-1,-1,-1), \gamma_{5}=i \gamma^{0} \gamma^{1} \gamma^{2} \gamma^{3}, \varepsilon^{0123}=+1$, and $\sigma^{\mu \nu}=\frac{i}{2}\left[\gamma^{\mu}, \gamma^{v}\right]$. To normalize the basis in a simple way, we choose the basis with a lower index as

$$
\Gamma_{B}=1 ; \gamma_{5} ; \gamma_{b} ; \gamma_{5} \gamma_{b} ; \sigma_{b_{1} b_{2}}\left(b_{1}<b_{2}\right)
$$

Note that the order of the factors for the axial vector has been deliberately flipped, so that the orthogonality condition $\operatorname{tr}\left(\Gamma^{A} \Gamma_{B}\right)=4 \delta_{B}^{A}$ holds uniformly.

An arbitrary $4 \times 4$ matrix $M$ can be expanded in the basis as $M=\sum_{A} x_{A} \Gamma^{A}$, with $4 x_{A}=$ $\operatorname{tr}\left(M \Gamma_{A}\right)$ by the orthogonality condition, i.e., $4 M=\sum_{A} \Gamma^{A} \operatorname{tr}\left(M \Gamma_{A}\right)$. The arbitrariness of $M$ implies $\sum_{C}\left(\Gamma_{C}\right)_{e f}\left(\Gamma^{C}\right)_{a d}=4 \delta_{e d} \delta_{a f}$, and multiplying it by $\left(\Gamma^{A}\right)_{f g}$ and summing over $f$ gives

$$
\sum_{C}\left(\Gamma_{C} \Gamma^{A}\right)_{e g}\left(\Gamma^{C}\right)_{a d}=4\left(\Gamma^{A}\right)_{a g} \delta_{e d}
$$

Taking the product of the above equality with another that is obtained from it by the substitutions egad $\rightarrow$ gecb and $C A \rightarrow D B$, and summing over ge yields finally

$$
4\left(\Gamma^{A}\right)_{a b}\left(\Gamma^{B}\right)_{c d}=\frac{1}{4} \sum_{C D} \operatorname{tr}\left(\Gamma_{C} \Gamma^{A} \Gamma_{D} \Gamma^{B}\right)\left(\Gamma^{C}\right)_{a d}\left(\Gamma^{D}\right)_{c b}
$$

which is essentially the most general Fierz identity. One point deserves special attention. In the sum over $C$ (similarly with $D$ ) the six independent components of the tensor are included only once; to recover the more convenient convention of Lorentz contraction we include a factor of $1 / 2$ when $C$ refers to the tensor term. Using the brief notations for the direct products of matrices, the above will be denoted as

$$
4 \Gamma^{A} \otimes \Gamma^{B}=\eta_{C} \eta_{D} \frac{1}{4} \operatorname{tr}\left(\Gamma_{C} \Gamma^{A} \Gamma_{D} \Gamma^{B}\right) \Gamma^{C} \odot \Gamma^{D}
$$

where $\eta_{C}=1 / 2$ when $C$ refers to the tensor and $\eta_{C}=1$ otherwise (and similarly for $\eta_{D}$ ). In terms of the spin-1/2 field operators the above means

$$
-4 \bar{\psi}_{1} \Gamma^{A} \psi_{2} \bar{\psi}_{3} \Gamma^{B} \psi_{4}=\eta_{C} \eta_{D} \frac{1}{4} \operatorname{tr}\left(\Gamma_{C} \Gamma^{A} \Gamma_{D} \Gamma^{B}\right) \bar{\psi}_{1} \Gamma^{C} \psi_{4} \bar{\psi}_{3} \Gamma^{D} \psi_{2}
$$

where the minus sign on the left hand side takes care of the Grassmannian nature of the fields and does not appear when the identities are applied to the spinor wavefunctions.

We record in what follows the explicit results upon working out the traces. There are only fifteen independent identities instead of twenty five; for instance, one can obtain the result for $\gamma_{5} \otimes 1$ from that for $1 \otimes \gamma_{5}$ by interchanging the two matrices in each product on both sides.

The products involving $\Gamma^{A}=1$ are found to be

$$
\begin{aligned}
4[1 \otimes 1] & =[1 \odot 1]+\left[\gamma_{5} \odot \gamma_{5}\right]+\left[\gamma^{c} \odot \gamma_{c}\right]-\left[\gamma^{c} \gamma_{5} \odot \gamma_{c} \gamma_{5}\right]+\frac{1}{2}\left[\sigma^{c_{1} c_{2}} \odot \sigma_{c_{1} c_{2}}\right] \\
4\left[1 \otimes \gamma_{5}\right] & =\left[1 \odot \gamma_{5}+\leftrightarrow\right]+\left[\gamma_{c} \gamma_{5} \odot \gamma^{c}-\leftrightarrow\right]+\frac{1}{2} i\left[\sigma^{c_{1} c_{2}} \odot \tilde{\sigma}_{c_{1} c_{2}}\right]
\end{aligned}
$$




$$
\begin{aligned}
4\left[1 \otimes \gamma^{b}\right]= & {\left[\left(1 \odot \gamma^{b}-\gamma_{c} \gamma_{5} \odot \tilde{\sigma}^{b c}\right)+\leftrightarrow\right]+\left[\left(\gamma_{5} \odot \gamma^{b} \gamma_{5}+i \gamma_{c} \odot \sigma^{c b}\right)-\leftrightarrow\right] } \\
4\left[1 \otimes \gamma^{b} \gamma_{5}\right]= & {\left[\left(1 \odot \gamma^{b} \gamma_{5}-\gamma_{c} \odot \tilde{\sigma}^{b c}\right)+\leftrightarrow\right]+\left[\left(\gamma_{5} \odot \gamma^{b}+i \gamma_{c} \gamma_{5} \odot \sigma^{c b}\right)-\leftrightarrow\right] } \\
4\left[1 \otimes \sigma^{b_{1} b_{2}}\right]= & {\left[\left(1 \odot \sigma^{b_{1} b_{2}}+i \gamma_{5} \odot \tilde{\sigma}^{b_{1} b_{2}}-\varepsilon^{b_{1} b_{2} c d} \gamma_{c} \odot \gamma_{d} \gamma_{5}\right)+\leftrightarrow\right] } \\
& +i\left[\left(\gamma^{b_{2}} \odot \gamma^{b_{1}}+\gamma^{b_{1}} \gamma_{5} \odot \gamma^{b_{2}} \gamma_{5}+\sigma^{b_{2} d} \odot \sigma_{d}^{b_{1}}\right)-\leftrightarrow\right]
\end{aligned}
$$

where $\leftrightarrow$ means the interchange of the two matrices in each direct product of the forgoing terms, and the shortcut $\tilde{\sigma}^{\mu v} \equiv \frac{1}{2} \varepsilon^{\mu v \alpha \beta} \sigma_{\alpha \beta}=-i \sigma^{\mu v} \gamma_{5}$ has been introduced.

The products involving $\Gamma^{A}=\gamma_{5}$ are

$$
\begin{aligned}
4\left[\gamma_{5} \otimes \gamma_{5}\right]= & {[1 \odot 1]+\left[\gamma_{5} \odot \gamma_{5}\right]-\left[\gamma^{c} \odot \gamma_{c}\right]+\left[\gamma^{c} \gamma_{5} \odot \gamma_{c} \gamma_{5}\right]+\frac{1}{2}\left[\sigma^{c_{1} c_{2}} \odot \sigma_{c_{1} c_{2}}\right] } \\
4\left[\gamma_{5} \otimes \gamma^{b}\right]= & {\left[\left(1 \odot \gamma^{b} \gamma_{5}+\gamma_{c} \odot \tilde{\sigma}^{b c}\right)-\leftrightarrow\right]+\left[\left(\gamma_{5} \odot \gamma^{b}+i \gamma_{c} \gamma_{5} \odot \sigma^{b c}\right)+\leftrightarrow\right] } \\
4\left[\gamma_{5} \otimes \gamma^{b} \gamma_{5}\right]= & {\left[\left(1 \odot \gamma^{b}+\gamma_{c} \gamma_{5} \odot \tilde{\sigma}^{b c}\right)-\leftrightarrow\right]+\left[\left(\gamma_{5} \odot \gamma^{b} \gamma_{5}+i \gamma_{c} \odot \sigma^{b c}\right)+\leftrightarrow\right] } \\
4\left[\gamma_{5} \otimes \sigma^{b_{1} b_{2}}\right]= & {\left[\left(i 1 \odot \tilde{\sigma}^{b_{1} b_{2}}+\gamma_{5} \odot \sigma^{b_{1} b_{2}}+i\left(\gamma^{b_{2}} \odot \gamma^{b_{1}} \gamma_{5}-b_{1} \leftrightarrow b_{2}\right)\right)+\leftrightarrow\right] } \\
& +\varepsilon^{b_{1} b_{2} c d}\left[-\gamma_{c} \odot \gamma_{d}+\gamma_{c} \gamma_{5} \odot \gamma_{d} \gamma_{5}+\sigma_{c e} \odot \sigma_{d}^{e}\right] \\
& +\left[\left(\tilde{\sigma}^{b_{1} d} \odot \sigma_{d}^{b_{2}}-b_{1} \leftrightarrow b_{2}\right)+\leftrightarrow\right]
\end{aligned}
$$

while the products involving $\Gamma^{A}=\gamma^{a}$ are

$$
\begin{aligned}
4\left[\gamma^{a} \otimes \gamma^{b}\right]= & {\left[\left(i 1 \odot \sigma^{a b}+\gamma_{5} \odot \tilde{\sigma}^{a b}+i \varepsilon^{a b c d} \gamma_{c} \odot \gamma_{d} \gamma_{5}\right)-\leftrightarrow\right] } \\
& +g^{a b}\left[1 \odot 1-\gamma_{5} \odot \gamma_{5}\right]+\left[\gamma^{a} \odot \gamma^{b}+\gamma^{b} \odot \gamma^{a}-g^{a b} \gamma^{c} \odot \gamma_{c}\right] \\
& +\left[\gamma^{a} \gamma_{5} \odot \gamma^{b} \gamma_{5}+\gamma^{b} \gamma_{5} \odot \gamma^{a} \gamma_{5}-g^{a b} \gamma^{c} \gamma_{5} \odot \gamma_{c} \gamma_{5}\right] \\
& +\frac{1}{2}\left[g^{a b} \sigma^{c_{1} c_{2}} \odot \sigma_{c_{1} c_{2}}-2 \sigma^{a c} \odot \sigma^{b}{ }_{c}-2 \sigma^{b c} \odot \sigma^{a}{ }_{c}\right] \\
4\left[\gamma^{a} \otimes \gamma^{b} \gamma_{5}\right]= & g^{a b}\left[\gamma_{5} \odot 1-\leftrightarrow\right]+i \varepsilon^{a b c d}\left[\gamma_{c} \odot \gamma_{d}+\gamma_{c} \gamma_{5} \odot \gamma_{d} \gamma_{5}\right] \\
& +\left[\left(\gamma^{a} \odot \gamma^{b} \gamma_{5}+\gamma^{b} \odot \gamma^{a} \gamma_{5}-g^{a b} \gamma^{c} \odot \gamma_{c} \gamma_{5}\right)+\leftrightarrow\right] \\
& +\left[\left(1 \odot \tilde{\sigma}^{a b}+i \gamma_{5} \odot \sigma^{a b}\right)+\leftrightarrow\right]+i \varepsilon^{a b c d} \sigma_{c e} \odot \sigma^{e}{ }_{d} \\
& +i\left[\sigma^{a}{ }_{c} \odot \tilde{\sigma}^{b c}-\leftrightarrow\right]-i\left[\sigma^{b}{ }_{c} \odot \tilde{\sigma}^{a c}+\leftrightarrow\right]+\frac{1}{2} i g^{a b} \sigma_{c_{1} c_{2}} \odot \tilde{\sigma}^{c_{1} c_{2}}
\end{aligned}
$$

and

$$
\begin{aligned}
& 4\left[\gamma^{a} \otimes \sigma^{b_{1} b_{2}}\right] \\
= & i\left[\left(g^{a b_{2}} 1 \odot \gamma^{b_{1}}-{ }^{b_{1}} \leftrightarrow{ }^{b_{2}}\right)-\leftrightarrow\right]+i\left[\left(g^{a b_{1}} \gamma_{5} \odot \gamma^{b_{2}} \gamma_{5}-{ }^{b_{1}} \leftrightarrow{ }^{b_{2}}\right)+\leftrightarrow\right] \\
& -\varepsilon^{a b_{1} b_{2} d}\left[1 \odot \gamma_{d} \gamma_{5}+\leftrightarrow\right]+\varepsilon^{a b_{1} b_{2} d}\left[\gamma_{5} \odot \gamma_{d}-\leftrightarrow\right]-i \varepsilon^{a b_{1} b_{2} d}\left[\gamma^{c} \gamma_{5} \odot \sigma_{c d}-\leftrightarrow\right] \\
& +\left[\left(g^{a b_{1}} \gamma^{c} \odot \sigma^{b_{2}}-g^{a b_{2}} \gamma^{c} \odot \sigma^{b_{1}}+\gamma^{a} \odot \sigma^{b_{1} b_{2}}+\gamma^{b_{1}} \odot \sigma^{a b_{2}}-\gamma^{b_{2}} \odot \sigma^{a b_{1}}\right)+\leftrightarrow\right] \\
& +i\left[\left(\varepsilon^{a b_{2} c d} \gamma_{c} \gamma_{5} \odot \sigma^{b_{1}}-{ }_{d}^{b_{1}} \leftrightarrow{ }^{b_{2}}\right)+\leftrightarrow\right]+i \varepsilon^{b_{1} b_{2} c d}\left[\gamma_{c} \gamma_{5} \odot \sigma^{a}{ }_{d}-\leftrightarrow\right] \\
& +i\left[\left(\left(g^{a b_{2}} \gamma_{c} \gamma_{5} \odot \tilde{\sigma}^{b_{1} c}+\gamma^{b_{2}} \gamma_{5} \odot \tilde{\sigma}^{a b_{1}}\right)-{ }^{b_{1}} \leftrightarrow{ }^{b_{2}}\right)+\leftrightarrow\right] \\
& +i\left[\gamma^{a} \gamma_{5} \odot \tilde{\sigma}^{b_{1} b_{2}}-\leftrightarrow\right]
\end{aligned}
$$


The products involving $\Gamma^{A}=\gamma^{a} \gamma_{5}$ are

$$
\begin{aligned}
4\left[\gamma^{a} \gamma_{5} \otimes \gamma^{b} \gamma_{5}\right]= & g^{a b}\left[-1 \odot 1+\gamma_{5} \odot \gamma_{5}\right]-\frac{1}{2} g^{a b}\left[\sigma^{c_{1} c_{2}} \odot \sigma_{c_{1} c_{2}}\right] \\
& +\left[\sigma^{a c} \odot \sigma^{b}+\leftrightarrow\right]+\left[\gamma^{a} \odot \gamma^{b}+\gamma^{b} \odot \gamma^{a}-g^{a b} \gamma^{c} \odot \gamma_{c}\right] \\
& +\left[\gamma^{a} \gamma_{5} \odot \gamma^{b} \gamma_{5}+\gamma^{b} \gamma_{5} \odot \gamma^{a} \gamma_{5}-g^{a b} \gamma^{c} \gamma_{5} \odot \gamma_{c} \gamma_{5}\right] \\
& -i\left[\left(1 \odot \sigma^{a b}-\gamma_{5} \odot \sigma^{a b} \gamma_{5}-\varepsilon^{a b c d} \gamma_{c} \odot \gamma_{d} \gamma_{5}\right)-\leftrightarrow\right]
\end{aligned}
$$

and

$$
\begin{aligned}
& 4\left(\gamma^{a} \gamma_{5} \otimes \sigma^{b_{1} b_{2}}\right) \\
= & -\varepsilon^{a b_{1} b_{2} c}\left[1 \odot \gamma_{c}+\leftrightarrow\right]+i\left[\left(g^{a b_{2}} 1 \odot \gamma^{b_{1}} \gamma_{5}-{ }^{b_{1}} \leftrightarrow{ }^{b_{2}}\right)-\leftrightarrow\right] \\
& +\varepsilon^{a b_{1} b_{2} c}\left[\gamma_{5} \odot \gamma_{c} \gamma_{5}-\leftrightarrow\right]+i\left[\left(g^{a b_{1}} \gamma_{5} \odot \gamma^{b_{2}}-{ }^{b_{1}} \leftrightarrow{ }^{b_{2}}\right)+\leftrightarrow\right] \\
& +i\left[\left(\varepsilon^{b_{1} b_{2} c d} \gamma_{c} \odot \sigma^{a d}-\varepsilon^{a b_{1} b_{2} d} \gamma^{c} \odot \sigma_{c d}\right)-\leftrightarrow\right]+i\left[\left(\varepsilon^{a b_{2} c d} \gamma_{c} \odot \sigma^{b_{1} d}-{ }^{b_{1}} \leftrightarrow{ }^{b_{2}}\right)+\leftrightarrow\right] \\
& +\left[\left(\left(g^{a b_{2}} \gamma_{c} \odot \sigma^{b_{1} c} \gamma_{5}+\gamma^{b_{2}} \odot \sigma^{a b_{1}} \gamma_{5}\right)-{ }^{b_{1}} \leftrightarrow{ }^{b_{2}}\right)+\leftrightarrow\right]+\left[\gamma^{a} \odot \sigma^{b_{1} b_{2}} \gamma_{5}-\leftrightarrow\right] \\
& +\left[\left(\left(g^{a b_{1}} \gamma_{c} \gamma_{5} \odot \sigma^{b_{2} c}+\gamma^{b_{1}} \gamma_{5} \odot \sigma^{a b_{2}}\right)-{ }^{b_{1}} \leftrightarrow{ }^{b_{2}}\right)+\leftrightarrow\right]+\left[\gamma^{a} \gamma_{5} \odot \sigma^{b_{1} b_{2}}+\leftrightarrow\right]
\end{aligned}
$$

Finally, the expression for the product $\sigma^{a_{1} a_{2}} \otimes \sigma^{b_{1} b_{2}}$ is very lengthy:

$$
\begin{aligned}
& 4\left(\sigma^{a_{1} a_{2}} \otimes \sigma^{b_{1} b_{2}}\right) \\
= & \left(g^{a_{1} b_{1}} g^{a_{2} b_{2}}-{ }^{b_{1}} \leftrightarrow{ }^{b_{2}}\right)\left[1 \odot 1+\gamma_{5} \odot \gamma_{5}+\gamma^{c} \odot \gamma_{c}-\gamma^{c} \gamma_{5} \odot \gamma_{c} \gamma_{5}+\frac{1}{2} \sigma^{c_{1} c_{2}} \odot \sigma_{c_{1} c_{2}}\right] \\
& +i \varepsilon^{a_{1} a_{2} b_{1} b_{2}}\left[1 \odot \gamma_{5}+\leftrightarrow\right]-i \varepsilon^{a_{1} a_{2} b_{1} b_{2}}\left[\gamma_{c} \odot \gamma^{c} \gamma_{5}-\leftrightarrow\right] \\
& +i\left[\left(\left(g^{a_{1} b_{1}} 1 \odot \sigma^{a_{2} b_{2}}+g^{a_{2} b_{2}} 1 \odot \sigma^{a_{1} b_{1}}\right)-{ }^{b_{1}} \leftrightarrow{ }^{b_{2}}\right)-\leftrightarrow\right] \\
& +\left[\left(\varepsilon^{a_{1} a_{2} b_{1} d} \gamma_{5} \odot \sigma^{b_{2}}-{ }^{b_{1}} \leftrightarrow{ }^{b_{2}}\right)+\leftrightarrow\right]+\left[\left(\varepsilon^{a_{2} b_{1} b_{2} d} \gamma_{5} \odot \sigma^{a_{1}}-{ }_{d}^{a_{1}} \leftrightarrow{ }^{a_{2}}\right)-\leftrightarrow\right] \\
& +\left[\left(\left(g^{a_{1} b_{1}} \gamma_{5} \odot \tilde{\sigma}^{a_{2} b_{2}}+g^{a_{2} b_{2}} \gamma_{5} \odot \tilde{\sigma}^{a_{1} b_{1}}\right)-{ }^{b_{1}} \leftrightarrow{ }^{b_{2}}\right)+\leftrightarrow\right] \\
& +\left[\left(\left(g^{a_{2} b_{1}} \gamma^{b_{2}} \odot \gamma^{a_{1}}+g^{a_{1} b_{2}} \gamma^{b_{1}} \odot \gamma^{a_{2}}\right)-{ }^{b_{1}} \leftrightarrow{ }^{b_{2}}\right)+\leftrightarrow\right] \\
& -\left[\left(\left(g^{a_{2} b_{1}} \gamma^{b_{2}} \gamma_{5} \odot \gamma^{a_{1}} \gamma_{5}+g^{a_{1} b_{2}} \gamma^{b_{1}} \gamma_{5} \odot \gamma^{a_{2}} \gamma_{5}\right)-{ }^{b_{1}} \leftrightarrow \leftrightarrow{ }^{b_{2}}\right)+\leftrightarrow\right] \\
& +i\left[\left(\varepsilon^{a_{1} a_{2} b_{2} c}\left(\gamma_{c} \odot \gamma^{b_{1}} \gamma_{5}-\gamma^{b_{1}} \odot \gamma_{c} \gamma_{5}\right)-{ }^{b_{1}} \leftrightarrow{ }^{b_{2}}\right)+\leftrightarrow\right] \\
& +i\left[\left(\varepsilon^{a_{1} b_{1} b_{2} c}\left(\gamma_{c} \odot \gamma^{a_{2}} \gamma_{5}+\gamma^{a_{2}} \odot \gamma_{c} \gamma_{5}\right)-{ }^{a_{1}} \leftrightarrow{ }^{a_{2}}\right)-\leftrightarrow\right] \\
& +i\left[\left(\left(g^{a_{1} b_{1}} \varepsilon^{a_{2} b_{2} c d}+g^{a_{2} b_{2}} \varepsilon^{a_{1} b_{1} c d}\right)-{ }^{b_{1}} \leftrightarrow{ }^{b_{2}}\right) \gamma_{c} \odot \gamma_{d} \gamma_{5}+\leftrightarrow\right] \\
& +\left[\left(\sigma^{a_{1} a_{2}} \odot \sigma^{b_{1} b_{2}}+\sigma^{a_{1} b_{1}} \odot \sigma^{a_{2} b_{2}}+\sigma^{b_{1} a_{2}} \odot \sigma^{a_{1} b_{2}}\right)+\leftrightarrow\right] \\
& +\left[\left(\left(g^{a_{1} b_{1}} \sigma^{c a_{2}} \odot \sigma^{b_{2}}+g^{a_{2} b_{2}} \sigma^{c b_{1}} \odot \sigma^{a_{1}}\right)-{ }^{a_{1}} \leftrightarrow{ }^{a_{2}}\right)+\leftrightarrow\right]
\end{aligned}
$$

The above identities are the most general ones for $4 \times 4$ matrices, and can reproduce as special cases the fully or partially Lorentz-contracted ones reported in the literature. For instance, the standard Lorentz-scalar Fierz identities are given by eqs (7/12) and eqs (16/19]21) upon contracting all Lorentz indices with the signature tensor, while the generalized Lorentzpseudoscalar identities listed in [3] are given by eq (8), eq (17) upon contraction by $g_{a b}$, and eq (21) upon contraction by $\varepsilon_{a_{1} a_{2} b_{1} b_{2}}$. 


\section{Identities for chiral spinors}

The Fierz identities are usually used to rearrange the fields in effective operators. Since the fermion fields in the standard model are chiral, those operators are naturally given in terms of chiral fields. It is thus desirable in this case to have the generalized identities projected onto various combinations of the two chiralities. There are two equivalent approaches to work them out. One can either choose a (partially) chiral basis, e.g., $P_{\mp}, \gamma^{a} P_{\mp}, \sigma^{a b}$, and proceed as in the last section, or obtain the identities by directly applying the projectors $P_{\mp}$ to the results recorded above. We have computed in both approaches and arrived at identical results. It is very nice to find that employing chiral fields significantly simplifies the identities.

We first introduce some notations. We use the shortcuts $\psi_{1 \mp}=P_{\mp} \psi_{1}$ and $\bar{\psi}_{1 \mp}=\left(\psi_{1 \mp}\right)^{\dagger} \gamma_{0}=$ $\bar{\psi}_{1} P_{ \pm}$. Here $\psi_{1}$ is a fermion field but can be spin-1/2 or spin-3/2 (or even higher). In the latter case $\psi_{1}$ implicitly carries a Lorentz index. We call $\bar{\psi}_{1} \Gamma^{A} \psi_{2 \mp} \bar{\psi}_{3} \Gamma^{B} \psi_{4 \mp}$ as chirality-diagonal and $\bar{\psi}_{1} \Gamma^{A} \psi_{2 \mp} \bar{\psi}_{3} \Gamma^{B} \psi_{4 \pm}$ as chirality-flipped. For a given pair of $\Gamma^{A, B}$, the chiralities of $\bar{\psi}_{1}$ and $\bar{\psi}_{3}$ are accordingly fixed and will be carried over to the Fierz-rearranged terms. This is natural and sufficient in practice since one cannot change the chirality of a field by applying an algebraic relation. In other words, the identities to be presented below apply to the spinor fields of definite chiralities.

We start with the simplest identity in eq (7). We project it out by attaching $P_{\mp}$ from right to the two matrices (identity in this case) in the product on the left-hand side, so that we will obtain a Fierz identity for $\bar{\psi}_{1 \pm} \psi_{2 \mp} \bar{\psi}_{3 \pm} \psi_{4 \mp}$, where the chirality of $\psi_{1}\left(\psi_{3}\right)$ is singled out by that of $\psi_{2}\left(\psi_{4}\right)$. One can see that the first two terms on the right-hand side become equal while the third and fourth terms are killed due to the mismatch in chirality. The result in terms of field operators is

$$
-4 \bar{\psi}_{1 \pm} \psi_{2 \mp} \bar{\psi}_{3 \pm} \psi_{4 \mp}=2 \bar{\psi}_{1 \pm} \psi_{4 \mp} \bar{\psi}_{3 \pm} \psi_{2 \mp}+\frac{1}{2} \bar{\psi}_{1 \pm} \sigma^{c_{1} c_{2}} \psi_{4 \mp} \bar{\psi}_{3 \pm} \sigma_{c_{1} c_{2}} \psi_{2 \mp}
$$

We will write the above as an algebraic relation:

$$
4\left[P_{\mp} \otimes P_{\mp}\right] \sim 2\left[P_{\mp} \odot P_{\mp}\right]+\frac{1}{2}\left[\sigma^{c_{1} c_{2}} P_{\mp} \odot \sigma_{c_{1} c_{2}} P_{\mp}\right]
$$

where the similarity symbol becomes equality when the spinor wavefunctions (field operators) are attached (with a minus sign on the left). In a similar manner, we can obtain the chiralityflipped identity by projecting eq (7) with $P_{\mp} \cdots P_{ \pm}$on its left side. Note that the projectors are interchanged on its right side, becoming $P_{ \pm} \cdots P_{\mp}$, so that the third and fourth terms become equal while all other three are killed:

$$
4\left[P_{\mp} \otimes P_{ \pm}\right] \sim 2\left[\gamma^{c} P_{ \pm} \odot \gamma_{c} P_{\mp}\right]
$$

The above two relations can also be obtained by starting from eq (8), which serves as a consistency check of the result in the standard basis. In so doing for the chirality-diagonal case, the relation (63) in Appendix is employed. The generalized Fierz identities to be listed in the following all pass similar consistency checks. 
For brevity, we first present the results and then make some brief comments on the derivation. The generalized Fierz identities are, in the chirality-diagonal case,

$$
\begin{aligned}
& 4\left[P_{\mp} \otimes P_{\mp}\right] \sim 2\left[P_{\mp} \odot P_{\mp}\right]+\frac{1}{2}\left[\sigma^{c_{1} c_{2}} P_{\mp} \odot \sigma_{c_{1} c_{2}} P_{\mp}\right] \\
& 4\left[P_{\mp} \otimes \gamma^{b} P_{\mp}\right] \sim 2\left[P_{\mp} \odot \gamma^{b} P_{\mp}\right]+2 i\left[\sigma^{b c} P_{\mp} \odot \gamma_{c} P_{\mp}\right] \\
& 4\left[P_{\mp} \otimes \sigma^{b_{1} b_{2}} P_{\mp}\right] \sim 2\left[P_{\mp} \odot \sigma^{b_{1} b_{2}} P_{\mp}+\leftrightarrow\right]+i\left[\sigma^{b_{2} d} P_{\mp} \odot \sigma_{d}^{b_{1}} P_{\mp}-\leftrightarrow\right] \\
& 4\left[\gamma^{a} P_{\mp} \otimes \gamma^{b} P_{\mp}\right] \sim 2\left[\gamma^{a} P_{\mp} \odot \gamma^{b} P_{\mp}+\gamma^{b} P_{\mp} \odot \gamma^{a} P_{\mp}-g^{a b} \gamma^{c} P_{\mp} \odot \gamma_{c} P_{\mp}\right] \\
& \mp 2 i \varepsilon^{a b c d} \gamma_{c} P_{\mp} \odot \gamma_{d} P_{\mp} \\
& 4\left[\gamma^{a} P_{\mp} \otimes \sigma^{b_{1} b_{2}} P_{\mp}\right] \sim 2 i\left[g^{a b_{1}} \gamma^{b_{2}} P_{\mp} \odot P_{\mp}-{ }^{b_{1}} \leftrightarrow{ }^{b_{2}}\right] \\
& \pm 2 \varepsilon^{a b_{1} b_{2} d} \gamma_{d} P_{\mp} \odot P_{\mp}+2 \gamma^{a} P_{\mp} \odot \sigma^{b_{1} b_{2}} P_{\mp} \\
& +2\left[\left(g^{a b_{1}} \gamma_{c} P_{\mp} \odot \sigma^{b_{2} c} P_{\mp}+\gamma^{b_{1}} P_{\mp} \odot \sigma^{a b_{2}} P_{\mp}\right)-{ }^{b_{1}} \leftrightarrow{ }^{b_{2}}\right] \\
& 4\left[\sigma^{a_{1} a_{2}} P_{\mp} \otimes \sigma^{b_{1} b_{2}} P_{\mp}\right] \sim \frac{1}{2}\left(g^{a_{1} b_{1}} g^{a_{2} b_{2}}-{ }^{b_{1}} \leftrightarrow{ }^{b_{2}}\right)\left[4 P_{\mp} \odot P_{\mp}-\sigma^{c_{1} c_{2}} P_{\mp} \odot \sigma_{c_{1} c_{2}} P_{\mp}\right] \\
& +\left[\sigma^{a_{1} a_{2}} P_{\mp} \odot \sigma^{b_{1} b_{2}} P_{\mp}+\leftrightarrow\right] \mp 2 i \varepsilon^{a_{1} a_{2} b_{1} b_{2}} P_{\mp} \odot P_{\mp} \\
& +2 i\left[P_{\mp} \odot\left(\left(g^{a_{1} b_{1}} \sigma^{a_{2} b_{2}}+g^{a_{2} b_{2}} \sigma^{a_{1} b_{1}}\right)-{ }^{b_{1}} \leftrightarrow{ }^{b_{2}}\right) P_{\mp}-\leftrightarrow\right] \\
& +\left[\left(\sigma^{a_{1} b_{1}} P_{\mp} \odot \sigma^{a_{2} b_{2}} P_{\mp}-{ }^{a_{1}} \leftrightarrow{ }^{a_{2}}\right)+\leftrightarrow\right]
\end{aligned}
$$

and in the chirality-flipped case,

$$
\begin{aligned}
& 4\left[P_{\mp} \otimes P_{ \pm}\right] \sim 2\left[\gamma^{c} P_{ \pm} \odot \gamma_{c} P_{\mp}\right] \\
& 4\left[P_{\mp} \otimes \gamma^{b} P_{ \pm}\right] \sim 2\left[\gamma^{b} P_{ \pm} \odot P_{\mp}\right]+2 i\left[\gamma_{c} P_{ \pm} \odot \sigma^{c b} P_{\mp}\right] \\
& 4\left[P_{\mp} \otimes \sigma^{b_{1} b_{2}} P_{ \pm}\right] \sim 2 i\left[\gamma^{b_{2}} P_{ \pm} \odot \gamma^{b_{1}} P_{\mp}-{ }^{b_{1}} \leftrightarrow{ }^{b_{2}}\right] \pm 2 \varepsilon^{b_{1} b_{2} c d}\left[\gamma_{c} P_{ \pm} \odot \gamma_{d} P_{\mp}\right] \\
& 4\left[\gamma^{a} P_{\mp} \otimes \gamma^{b} P_{ \pm}\right] \sim 2 g^{a b}\left[P_{ \pm} \odot P_{\mp}\right]-\left[\sigma^{a c} P_{ \pm} \odot \sigma^{b}{ }_{c} P_{\mp}+{ }^{a} \leftrightarrow{ }^{b}\right] \\
& +2 i\left[P_{ \pm} \odot \sigma^{a b} P_{\mp}-\leftrightarrow\right] \\
& 4\left[\gamma^{a} P_{\mp} \otimes \sigma^{b_{1} b_{2}} P_{ \pm}\right] \sim 2 i\left[g^{a b_{2}} P_{ \pm} \odot \gamma^{b_{1}} P_{\mp}-b_{1} \leftrightarrow{ }^{b_{2}}\right] \\
& \pm 2 \varepsilon^{a b_{1} b_{2} d} P_{ \pm} \odot \gamma_{d} P_{\mp}+2 \sigma^{b_{1} b_{2}} P_{ \pm} \odot \gamma^{a} P_{\mp} \\
& +2\left[\left(g^{a b_{1}} \sigma_{c}^{b_{2}} P_{ \pm} \odot \gamma^{c} P_{\mp}+\sigma^{a b_{2}} P_{ \pm} \odot \gamma^{b_{1}} P_{\mp}\right)-{ }^{b_{1}} \leftrightarrow{ }^{b_{2}}\right] \\
& 4\left[\sigma^{a_{1} a_{2}} P_{\mp} \otimes \sigma^{b_{1} b_{2}} P_{ \pm}\right] \sim 2\left[\left(\left(g^{a_{2} b_{1}} \gamma^{b_{2}} P_{ \pm} \odot \gamma^{a_{1}} P_{\mp}+g^{a_{1} b_{2}} \gamma^{b_{1}} P_{ \pm} \odot \gamma^{a_{2}} P_{\mp}\right)\right.\right. \\
& \left.\left.-{ }^{b_{1}} \leftrightarrow{ }^{b_{2}}\right)+\leftrightarrow\right]+2\left(g^{a_{1} b_{1}} g^{a_{2} b_{2}}-g^{a_{1} b_{2}} g^{a_{2} b_{1}}\right) \gamma^{c} P_{ \pm} \odot \gamma_{c} P_{\mp} \\
& \mp 2 i\left(\varepsilon^{a_{1} a_{2} b_{2} c} g^{b_{1} d}-\varepsilon^{a_{1} a_{2} b_{1} c} g^{b_{2} d}\right. \\
& \left.+\varepsilon^{a_{1} b_{1} b_{2} d} g^{a_{2} c}-\varepsilon^{a_{2} b_{1} b_{2} d} g^{a_{1} c}\right) \gamma_{c} P_{ \pm} \odot \gamma_{d} P_{\mp}
\end{aligned}
$$

Note that the interchange of matrices $\leftrightarrow$ does not act on the chiral projectors; for instance, the second term in the last square brackets of eq (32) reads, $-\sigma^{a b} P_{ \pm} \odot P_{\mp}$.

In checking eq (32) obtained from eq (16) against the one from eq (17) or eq (19), eqs (68 69 70) in Appendix are used. In deriving eq (28), eqs (66/71) are applied, while eqs (64)65) are employed to cast eq (34) in the displayed form. Eq (64) is also useful in simplifying eqs (27/33), while eq (66) is used to recast eq (27) in a form similar to eq (33). Finally, eq (72) is used in checking eq (25) obtained from eq (11) against that from eq (15). 
We illustrate the above results by a few examples of sextuple forms in spin- $1 / 2$ fields. The first one concerns the operators $A_{\mp}=\bar{\psi}_{1 \pm} \psi_{2 \mp} \bar{\psi}_{3 \pm} \psi_{4 \mp} \bar{\psi}_{5 \pm} \psi_{6 \mp}$. They have the same Lorentz structure as the instanton-induced six-quark interaction. We will not try to transform them to a form that would be relevant to the study of color-superconductivity [8], which involves rearrangements of the barred and unbarred fields with charge conjugation as well as nontrivial structures in flavor and color spaces. Instead, we are content with transforming them to a form with $\psi_{2,4,6}$ replaced by $\psi_{4,6,2}$. This can be accomplished by twice applications of eqs (23, 28$)$. Although the procedures are not unique, the end result must be the same. For instance, we may first apply eq (23) to $\bar{\psi}_{1 \pm} \psi_{2 \mp} \bar{\psi}_{3 \pm} \psi_{4 \mp}$ and then eqs (23/25) to $\bar{\psi}_{3 \pm} \Gamma^{A} \psi_{2 \mp} \bar{\psi}_{5 \pm} \psi_{6 \mp}$. The Grassmannian minus signs are cancelled in the final result:

$$
\begin{aligned}
16 A_{\mp}= & 4 \bar{\psi}_{1 \pm} \psi_{4 \mp} \bar{\psi}_{3 \pm} \psi_{6 \mp} \bar{\psi}_{5 \pm} \psi_{2 \mp}+i \bar{\psi}_{1 \pm} \sigma_{d c} \psi_{4 \mp} \bar{\psi}_{3 \pm} \sigma_{e}^{c} \psi_{6 \mp} \bar{\psi}_{5 \pm} \sigma^{e d} \psi_{2 \mp} \\
& +\bar{\psi}_{1 \pm} \psi_{4 \mp} \bar{\psi}_{3 \pm} \sigma^{c d} \psi_{6 \mp} \bar{\psi}_{5 \pm} \sigma_{c d} \psi_{2 \mp}+\bar{\psi}_{1 \pm} \sigma_{c d} \psi_{4 \mp} \bar{\psi}_{3 \pm} \sigma^{c d} \psi_{6 \mp} \bar{\psi}_{5 \pm} \psi_{2 \mp} \\
& +\bar{\psi}_{1 \pm} \sigma_{c d} \psi_{4 \mp} \bar{\psi}_{3 \pm} \psi_{6 \mp} \bar{\psi}_{5 \pm} \sigma^{c d} \psi_{2 \mp}
\end{aligned}
$$

Consider next the operators with a contracted vector, $B_{\mp}=\bar{\psi}_{1 \mp} \gamma^{a} \psi_{2 \mp} \bar{\psi}_{3 \mp} \gamma_{a} \psi_{4 \mp} \bar{\psi}_{5 \pm} \psi_{6 \mp}$, which can be Fierz-rewritten as

$$
2 B_{\mp}=-\bar{\psi}_{1 \mp} \gamma^{a} \psi_{4 \mp}\left[\bar{\psi}_{3 \mp} \gamma_{a} \psi_{6 \mp} \bar{\psi}_{5 \pm} \psi_{2 \mp}+i \bar{\psi}_{3 \mp} \gamma^{b} \psi_{6 \mp} \bar{\psi}_{5 \pm} \sigma_{a b} \psi_{2 \mp}\right]
$$

Finally, compare a pair of operators that differ by chirality in one bilinear,

$$
\begin{aligned}
& C_{\mp}=\bar{\psi}_{1 \mp} \gamma^{a} \psi_{2 \mp} \bar{\psi}_{3 \mp} \gamma^{b} \psi_{4 \mp} \bar{\psi}_{5 \pm} \sigma_{a b} \psi_{6 \mp} \\
& D_{\mp}=\bar{\psi}_{1 \mp} \gamma^{a} \psi_{2 \mp} \bar{\psi}_{3 \pm} \gamma^{b} \psi_{4 \pm} \bar{\psi}_{5 \pm} \sigma_{a b} \psi_{6 \mp}
\end{aligned}
$$

It is easier to first apply eqs (26/32) and keep terms antisymmetric in $a$ and $b$, and then use eqs 27/3134) to obtain

$$
\begin{aligned}
4 C_{\mp}= & \bar{\psi}_{1 \mp} \gamma_{c} P_{\mp} \psi_{4 \mp}\left[-6 i \bar{\psi}_{3 \mp} \gamma^{c} \psi_{6 \mp} \bar{\psi}_{5 \pm} \psi_{2 \mp}-2 \bar{\psi}_{3 \mp} \gamma_{d} \psi_{6 \mp} \bar{\psi}_{5 \pm} \sigma^{c d} \psi_{2 \mp}\right] \\
4 D_{\mp}= & i \bar{\psi}_{1 \mp} \psi_{4 \pm}\left[12 \bar{\psi}_{3 \pm} \psi_{6 \mp} \bar{\psi}_{5 \pm} \psi_{2 \mp}-4 \bar{\psi}_{3 \pm} \sigma_{a b} \psi_{6 \mp} \bar{\psi}_{5 \pm} \sigma^{a b} \psi_{2 \mp}\right] \\
& -\bar{\psi}_{1 \mp} \sigma^{a b} \psi_{4 \pm} \bar{\psi}_{3 \pm} \sigma_{b d} \psi_{6 \mp} \bar{\psi}_{5 \pm} \sigma_{a}^{d} \psi_{2 \mp}
\end{aligned}
$$

where eqs (63,68) have been used.

\section{Applications to spin-3/2 particles}

The quantum field of a spin-3/2 particle can be described by the Rarita-Schwinger (RS) field [12], $\Psi_{\mu}$. It is a vector-spinor that has the mixed transformation properties of a Dirac field and a vector field under Lorentz transformations. A free field of mass $M$ satisfies the Dirac equation, $(i \not \partial-M) \Psi_{\mu}=0$, with the constraint

$$
\gamma^{\mu} \Psi_{\mu}=0
$$

from which follows the relation $\partial^{\mu} \Psi_{\mu}=0$. For our purpose of constructing dimension-six four-fermion effective operators without a derivative, only the constraint (41) and its conjugate 
$\bar{\Psi}_{\mu} \gamma^{\mu}=0$ are relevant. In the following, we will construct those operators involving up to three factors of the RS fields that may be relevant to the phenomenology of the standard model particles, and when necessary verify their independence using the Fierz identities established in the last section.

We start with the operators containing a single RS field, $\Psi_{\mu}$, and three spin-1/2 chiral fields, $\psi_{1 \mp}, \psi_{2 \mp}, \psi_{3 \mp}$, i.e., of the types:

$$
\begin{aligned}
X_{\mp}^{\mathrm{d}} & =\bar{\psi}_{1} \Gamma^{A} P_{\mp} \psi_{2} \bar{\psi}_{3} \Gamma^{B} P_{\mp} \Psi_{\mu} \\
X_{ \pm}^{\mathrm{f}} & =\bar{\psi}_{1} \Gamma^{A} P_{\mp} \psi_{2} \bar{\psi}_{3} \Gamma^{B} P_{ \pm} \Psi_{\mu}
\end{aligned}
$$

where all Lorentz indices are to be properly contracted. Allocating the basis matrices to both $\Gamma^{A}$ and $\Gamma^{B}$ with an odd number of Lorentz indices yields the possibilities, $1 \otimes \gamma^{b}, \gamma^{a} \otimes 1, \gamma^{a} \otimes$ $\sigma^{b_{1} b_{2}}, \sigma^{a_{1} a_{2}} \otimes \gamma^{b}$, where $\gamma_{5}$ is excluded due to the presence of $P_{\mp}$. Now we contract the above indices with $\Psi_{\mu}$. It is not necessary either to employ the Levi-Civita tensor since, e.g., $\varepsilon^{a_{1} a_{2} c d} \sigma_{a_{1} a_{2}} P_{\mp}=2 \tilde{\sigma}^{c d} P_{\mp}= \pm 2 i \sigma^{c d} P_{\mp}$ using eq (63), which does not yield a new form. The first form is killed by eq (41). The third one is removed as redundant, since $\sigma_{a \mu} P_{\mp} \Psi^{\mu}=$ $i\left(\gamma_{a} \gamma_{\mu}-g_{a \mu}\right) P_{\mp} \Psi^{\mu}=-i P_{\mp} \Psi_{a}$, which is the second form in the list. The list is thus shortened to $\gamma^{\mu} \otimes 1, \sigma^{\mu v} \otimes \gamma_{v}$, corresponding to the operators

$$
\begin{aligned}
& X_{\mp}^{\mathrm{d}}=\bar{\psi}_{1} \gamma^{\mu} P_{\mp} \psi_{2} \bar{\psi}_{3} P_{\mp} \Psi_{\mu}, \bar{\psi}_{1} \sigma^{\mu v} P_{\mp} \psi_{2} \bar{\psi}_{3} \gamma_{v} P_{\mp} \Psi_{\mu} \\
& X_{ \pm}^{\mathrm{f}}=\bar{\psi}_{1} \gamma^{\mu} P_{\mp} \psi_{2} \bar{\psi}_{3} P_{ \pm} \Psi_{\mu}, \bar{\psi}_{1} \sigma^{\mu v} P_{\mp} \psi_{2} \bar{\psi}_{3} \gamma_{v} P_{ \pm} \Psi_{\mu}
\end{aligned}
$$

With the help of the identities in section 3 it can be verified that they are complete and independent, i.e., that one cannot get lesser or more operators by interchanging the roles of $\psi_{2}$ and $\Psi_{\mu}$ (or equivalently $\psi_{1}$ and $\psi_{3}$ ). Consider for instance the operators obtained from the second pair in $X_{\mp}^{\mathrm{d}}$ by $\bar{\psi}_{1} \leftrightarrow \bar{\psi}_{3}$. Using eq (27) upon contracting a pair of indices

$$
\begin{aligned}
& 4\left[\gamma_{v} P_{\mp} \otimes \sigma^{\mu v} P_{\mp}\right] \\
\sim & 2 i(-3) \gamma^{\mu} P_{\mp} \odot P_{\mp}+2 \gamma_{v} P_{\mp} \odot \sigma^{\mu v} P_{\mp} \odot \pm 2 i \varepsilon^{v \mu \alpha \beta} \gamma_{\alpha} P_{\mp} \odot \sigma_{v \beta} P_{\mp} \\
= & 2 i(-3) \gamma^{\mu} P_{\mp} \odot P_{\mp}-2 \gamma_{v} P_{\mp} \odot \sigma^{\mu v} P_{\mp}
\end{aligned}
$$

where eq (63) is used in the last term, we have upon applying the constraint (41)

$$
-4 \bar{\psi}_{1} \gamma_{v} P_{\mp} \Psi_{\mu} \bar{\psi}_{3} \sigma^{\mu v} P_{\mp} \psi_{2}=-8 i \bar{\psi}_{1} \gamma^{\mu} P_{\mp} \psi_{2} \bar{\psi}_{3} P_{\mp} \Psi_{\mu}
$$

which is indeed the first pair in $X_{\mp}^{\mathrm{d}}$.

The operators involving two spin-1/2 and two spin-3/2 fields are classified into two classes. In the first class we can write in either the format

$$
\text { (Ia) } \begin{aligned}
& Y_{\mp}^{(\text {Ia }) \mathrm{d}}=\bar{\Psi}_{1 \mu} \Gamma^{A} P_{\mp} \Psi_{2 \nu} \bar{\psi}_{1} \Gamma^{B} P_{\mp} \psi_{2} \\
& Y_{ \pm}^{(\mathrm{Ia}) \mathrm{f}}=\bar{\Psi}_{1 \mu} \Gamma^{A} P_{\mp} \Psi_{2 \nu} \bar{\psi}_{1} \Gamma^{B} P_{ \pm} \psi_{2}
\end{aligned}
$$

or its Fierz-transformed one

$$
\text { (Ib) } \begin{aligned}
& Y_{\mp}^{(\mathrm{Ib}) \mathrm{d}}=\bar{\Psi}_{1 \mu} \Gamma^{A} P_{\mp} \psi_{2} \bar{\psi}_{1} \Gamma^{B} P_{\mp} \Psi_{2 v} \\
& Y_{ \pm}^{(\mathrm{Ib}) \mathrm{f}}=\bar{\Psi}_{1 \mu} \Gamma^{A} P_{\mp} \psi_{2} \bar{\psi}_{1} \Gamma^{B} P_{ \pm} \Psi_{2 v}
\end{aligned}
$$


We keep both for the purpose of redundancy check. In the second class, we have

$$
\text { (II) } \begin{aligned}
& Y_{\mp}^{(\mathrm{II}) \mathrm{d}}=\bar{\psi}_{1} \Gamma^{A} P_{\mp} \Psi_{1 \mu} \bar{\psi}_{2} \Gamma^{B} P_{\mp} \Psi_{2 v} \\
& Y_{ \pm}^{(\mathrm{II}) \mathrm{f}}=\bar{\psi}_{1} \Gamma^{A} P_{\mp} \Psi_{1 \mu} \bar{\psi}_{2} \Gamma^{B} P_{ \pm} \Psi_{2 v}
\end{aligned}
$$

Note that the operators in class (II) violate any additive quantum number carried by the $\psi$ fields, e.g., the lepton or baryon number, or by the $\Psi_{\mu}$ fields. The pair of $\Gamma^{A, B}$ should have an even number of indices, $1 \otimes 1 ; \gamma^{a} \otimes \gamma^{b} ; 1 \otimes \sigma^{b_{1} b_{2}}, \sigma^{a_{1} a_{2}} \otimes 1 ; \sigma^{a_{1} a_{2}} \otimes \sigma^{b_{1} b_{2}}$, to be contracted with the field operators.

Consider first class (Ia). The possible forms without involving a $\sigma$ are

$$
\begin{aligned}
& g^{\mu v} P_{\mp} \otimes P_{\mp} ; g^{\mu v} \gamma^{a} P_{\mp} \otimes \gamma_{a} P_{\mp}, \varepsilon^{\mu v a b} \gamma_{a} P_{\mp} \otimes \gamma_{b} P_{\mp} \\
& g^{\mu v} P_{\mp} \otimes P_{ \pm} ; g^{\mu v} \gamma^{a} P_{\mp} \otimes \gamma_{a} P_{ \pm}, \varepsilon^{\mu v a b} \gamma_{a} P_{\mp} \otimes \gamma_{b} P_{ \pm}
\end{aligned}
$$

Due to eq (63), the possible forms containing one $\sigma$ are restricted to be

$$
\begin{aligned}
& P_{\mp} \otimes \sigma_{\mu \nu} P_{\mp} \\
& P_{\mp} \otimes \sigma_{\mu \nu} P_{ \pm}
\end{aligned}
$$

With two $\sigma$ 's, the completely self-contracted ones are

$$
g^{\mu v} \sigma^{a_{1} a_{2}} P_{\mp} \otimes \sigma_{a_{1} a_{2}} P_{\mp}
$$

whose chirality-flipped counterparts, $g^{\mu v} \sigma^{a_{1} a_{2}} P_{\mp} \otimes \sigma_{a_{1} a_{2}} P_{ \pm}$, vanish by eq (68). The terms with once self-contracted $\sigma$ 's are either reducible to those with one $\sigma$ in the chirality-diagonal case, e.g, $\sigma_{c}^{\mu}{ }_{c} P_{\mp} \otimes \sigma^{v c} P_{\mp}$ and $\varepsilon^{\mu v a b} \sigma_{a}^{c} P_{\mp} \otimes \sigma_{b c} P_{\mp}$ with the help of eqs (41172), or simply vanish by eqs 68169) in the chirality-flipped case. It thus appears that $Y_{\mp}^{(\mathrm{Ia}) \mathrm{d}}$ have five forms and $Y_{ \pm}^{(\mathrm{Ia}) \mathrm{f}}$ have four. A similar analysis shows that class (Ib) has the same number of forms:

$$
\begin{aligned}
& g^{\mu v} P_{\mp} \otimes P_{\mp} ; g^{\mu v} \gamma^{a} P_{\mp} \otimes \gamma_{a} P_{\mp}, \gamma^{v} P_{\mp} \otimes \gamma^{\mu} P_{\mp}, \varepsilon^{\mu v a b} \gamma_{a} P_{\mp} \otimes \gamma_{b} P_{\mp} ; g^{\mu v} \sigma^{a b} P_{\mp} \otimes \sigma_{a b} P_{\mp} \\
& g^{\mu v} P_{\mp} \otimes P_{ \pm} ; g^{\mu v} \gamma^{a} P_{\mp} \otimes \gamma_{a} P_{ \pm}, \gamma^{v} P_{\mp} \otimes \gamma^{\mu} P_{ \pm}, \varepsilon^{\mu v a b} \gamma_{a} P_{\mp} \otimes \gamma_{b} P_{ \pm}
\end{aligned}
$$

But the lists for class (Ia) and (Ib) are actually redundant. To see this, we can start from either (Ia) or (Ib). Starting from (Ia) (i.e., $\otimes$ for $(\mathrm{Ia})$ and $\odot$ for $(\mathrm{Ib}))$ and using eqs $(26 / 32 \sqrt[63]{ })$, we have

$$
\begin{aligned}
& 4\left[\gamma_{a} P_{\mp} \otimes \gamma_{b} P_{\mp}\right] \varepsilon^{\mu v a b} \sim \pm 4 i\left[\gamma^{\mu} P_{\mp} \odot \gamma^{v} P_{\mp}-\gamma^{v} P_{\mp} \odot \gamma^{\mu} P_{\mp}\right] \\
& 4\left[\gamma_{a} P_{\mp} \otimes \gamma_{b} P_{ \pm}\right] \varepsilon^{\mu v a b} \sim \pm 4 i\left[P_{ \pm} \odot \sigma^{\mu v} P_{\mp}+\sigma^{\mu v} P_{ \pm} \odot P_{\mp}\right]
\end{aligned}
$$

The first term in eq (53) is killed by eq (41) while the second term remains in the list of (Ib). Both terms in eq (54) reduce to $g^{\mu v} P_{ \pm} \odot P_{\mp}$ by eq (41). The above identities also apply when starting from $(\mathrm{Ib})$ (now $\otimes$ for $(\mathrm{Ib})$ and $\odot$ for $(\mathrm{Ia})$ ). Then, both terms in eq (53) are killed, while the first term in eq (54) remains and the second term reduces to $g^{\mu v} P_{ \pm} \odot P_{\mp}$. The conclusion from this is that $\gamma_{a} P_{\mp} \otimes \gamma_{b} P_{\mp} \varepsilon^{\mu v a b}$ and $\gamma_{a} P_{\mp} \otimes \gamma_{b} P_{ \pm} \varepsilon^{\mu v a b}$ can be simultaneously removed from 
the list in both (Ia) and (Ib). This result conforms to the list of fourteen independent operators in [11] in the standard basis. Finally, the analysis and result for (Ib) apply to class (II) as well.

We finally come to the operators involving three spin-3/2 and one spin-1/2 fields:

$$
\begin{aligned}
& Z_{\mp}^{\mathrm{d}}=\bar{\Psi}_{1 \alpha} \Gamma^{A} P_{\mp} \Psi_{2 \beta} \bar{\psi} \Gamma^{B} P_{\mp} \Psi_{3 \gamma} \\
& Z_{ \pm}^{\mathrm{f}}=\bar{\Psi}_{1 \alpha} \Gamma^{A} P_{\mp} \Psi_{2 \beta} \bar{\psi} \Gamma^{B} P_{ \pm} \Psi_{3 \gamma}
\end{aligned}
$$

The pair of $\Gamma^{A, B}$ with an odd number of indices includes $\gamma^{a} \otimes 1,1 \otimes \gamma^{b} ; \gamma^{a} \otimes \sigma^{b_{1} b_{2}}, \sigma^{a_{1} a_{2}} \otimes \gamma^{b}$, and thus the possible forms for both $Z_{\mp}^{\mathrm{d}, \mathrm{f}}$ are

$$
\begin{aligned}
& g^{\alpha \beta} \gamma^{\gamma} \otimes 1, \varepsilon^{\alpha \beta \gamma a} \gamma_{a} \otimes 1 \\
& g^{\alpha \gamma} 1 \otimes \gamma^{\beta}, g^{\beta \gamma} 1 \otimes \gamma^{\alpha}, \varepsilon^{\alpha \beta \gamma b} 1 \otimes \gamma_{b} \\
& \gamma^{\gamma} \otimes \sigma^{\alpha \beta}, g^{\beta \gamma} \gamma_{a} \otimes \sigma^{a \alpha}, g^{\alpha \gamma} \gamma_{a} \otimes \sigma^{a \beta} \\
& \varepsilon^{\alpha \beta \gamma b} \gamma^{a} \otimes \sigma_{a b}, \varepsilon^{\alpha \gamma a b} \gamma_{a} \otimes \sigma_{b}^{\beta}, \varepsilon^{\beta \gamma a b} \gamma_{a} \otimes \sigma_{b}^{\alpha} \\
& g^{\alpha \beta} \sigma^{\gamma b} \otimes \gamma_{b}, \varepsilon^{\alpha \beta \gamma a} \sigma_{a b} \otimes \gamma^{b}, \varepsilon^{\alpha \beta a b} \sigma_{a}^{\gamma} \otimes \gamma_{b}
\end{aligned}
$$

The last term in eqs (57/58) and the last two terms in eq (60) can be removed as redundant using eqs (67/41). For the first term in eq (60) and the second term in eq (61), using antisymmetry in $a, b$ and eq (64), we have

$$
\varepsilon^{\alpha \beta \gamma a} \gamma^{b}-\varepsilon^{\alpha \beta \gamma b} \gamma^{a}=-\varepsilon^{\beta \gamma a b} \gamma^{\alpha}-\varepsilon^{\gamma a b \alpha} \gamma^{\beta}-\varepsilon^{a b \alpha \beta} \gamma^{\gamma}
$$

which will transform $\sigma_{a b}$ into $\tilde{\sigma}^{\beta \gamma}$ etc and thus can be dropped. Finally, the last term in eq (61) can be recast using eq (66) as, for both chirality-diagonal and -flipped cases,

$$
\varepsilon^{\alpha \beta a b} \sigma_{a}^{\gamma} P_{\mp} \otimes \gamma_{b}= \pm i\left(g^{\beta \gamma} \sigma^{\alpha b}-g^{\alpha \gamma} \sigma^{\beta b}-g^{b \gamma} \sigma^{\alpha \beta}\right) P_{\mp} \otimes \gamma_{b}
$$

where the first two terms are reducible to those already covered while the third vanishes by eq (41). In summary, the list has been reduced for both $Z_{\mp}^{\mathrm{d}, \mathrm{f}}$ to

$$
\begin{aligned}
& g^{\alpha \beta} \gamma^{\gamma} \otimes 1, g^{\alpha \gamma} 1 \otimes \gamma^{\beta}, g^{\beta \gamma} 1 \otimes \gamma^{\alpha} \\
& \gamma^{\gamma} \otimes \sigma^{\alpha \beta}, g^{\beta \gamma} \gamma_{a} \otimes \sigma^{a \alpha}, g^{\alpha \gamma} \gamma_{a} \otimes \sigma^{a \beta}, g^{\alpha \beta} \sigma^{\gamma b} \otimes \gamma_{b}
\end{aligned}
$$

which are obviously independent as are explicitly verified by the generalized identities.

\section{Summary}

We have studied the general Fierz identities for all possible direct products of non-contracted Dirac matrices both in the standard basis and for chiral fields. The identities are helpful in rearranging fields in effective four-fermion operators involving derivatives and in sextuple or higher operators. They can also be applied to effective interactions involving higher-spin fermions. We have illustrated this by considering all dimension-six four-fermion operators that involve one to three spin-3/2 fields, and used the identities to check their independency. These operators 
could be relevant to the phenomenological study of baryons with a higher spin and of the recent interest in massive spin-3/2 particles as a possible candidate for dark matter.

\section{Acknowledgement}

This work was supported in part by the grant NSFC-11025525 and by the Fundamental Research Funds for the Central Universities No.65030021.

\section{Appendix: some useful relations}

We list below some algebraic relations that were employed in deriving and simplifying the generalized Fierz identities. The most often used is the basic relation

$$
\tilde{\sigma}^{\mu v} P_{\mp}= \pm i \sigma^{\mu v} P_{\mp}
$$

where $\tilde{\sigma}^{\mu v}=(1 / 2) \varepsilon^{\mu v \alpha \beta} \sigma_{\alpha \beta}$. In four-dimensions, we have

$$
p^{a} \varepsilon^{b c d e}+p^{b} \varepsilon^{c d e a}+p^{c} \varepsilon^{d e a b}+p^{d} \varepsilon^{e a b c}+p^{e} \varepsilon^{a b c d}=0
$$

where $p$ is an arbitrary four-component quantity, e.g., the gamma matrix, and

$$
\begin{aligned}
& 2\left(g^{a_{1} b_{1}} \varepsilon^{a_{2} b_{2} c d}-g^{a_{1} b_{2}} \varepsilon^{a_{2} b_{1} c d}-g^{a_{2} b_{1}} \varepsilon^{a_{1} b_{2} c d}+g^{a_{2} b_{2}} \varepsilon^{a_{1} b_{1} c d}\right) \\
=+ & +\left[\left(g^{b_{1} c} \varepsilon^{a_{1} a_{2} b_{2} d}-g^{b_{1} d} \varepsilon^{a_{1} a_{2} b_{2} c}\right)-\left(g^{b_{2} c} \varepsilon^{a_{1} a_{2} b_{1} d}-g^{b_{2} d} \varepsilon^{a_{1} a_{2} b_{1} c}\right)\right] \\
- & {\left[\left(g^{a_{1} c} \varepsilon^{b_{1} b_{2} a_{2} d}-g^{a_{1} d} \varepsilon^{b_{1} b_{2} a_{2} c}\right)-\left(g^{a_{2} c} \varepsilon^{b_{1} b_{2} a_{1} d}-g^{a_{2} d} \varepsilon^{b_{1} b_{2} a_{1} c}\right)\right] }
\end{aligned}
$$

which has been constructed by considering all possible six-index constant tensors made of one signature and one Levi-Civita tensor with specific symmetries in the indices. Using eq (63) and once-contracted two $\varepsilon$ 's, $\varepsilon^{a_{1} a_{2} b_{1} d} \varepsilon_{d c_{1} c_{2} b_{2}}$, one derives

$$
\mp \varepsilon^{a_{1} a_{2} b_{1} d} \sigma_{d}^{b_{2}} P_{\mp}=i\left(g^{a_{2} b_{2}} \sigma^{a_{1} b_{1}}-g^{a_{1} b_{2}} \sigma^{a_{2} b_{1}}-g^{b_{1} b_{2}} \sigma^{a_{1} a_{2}}\right) P_{\mp}
$$

Finally, using the well-known relation

$$
\gamma^{\alpha} \gamma^{\beta} \gamma^{\gamma}=\left(g^{\alpha \beta} \gamma^{\gamma}+g^{\beta \gamma} \gamma^{\alpha}-g^{\gamma \alpha} \gamma^{\beta}\right)-i \varepsilon_{\mu}^{\alpha \beta \gamma} \gamma^{\mu} \gamma_{5}
$$

the following result is obtained

$$
\varepsilon^{\alpha \beta \gamma a} \gamma_{a} P_{\mp}=\mp i\left(\gamma^{\alpha} \gamma^{\gamma} \gamma^{\beta}-g^{\alpha \gamma} \gamma^{\beta}-g^{\gamma \beta} \gamma^{\alpha}+g^{\alpha \beta} \gamma^{\gamma}\right) P_{\mp}
$$

which was used in section 4 to remove redundant operators.

Now we list some relations employed in simplifying operators and Fierz identities. The chirality-flipped ones are

$$
\begin{aligned}
& \sigma^{a b} P_{ \pm} \odot \sigma_{a b} P_{\mp}=0 \\
& \sigma^{a c} P_{ \pm} \odot \sigma^{b}{ }_{c} P_{\mp}=\sigma^{b c} P_{ \pm} \odot \sigma^{a}{ }_{c} P_{\mp} \\
& \varepsilon^{a b c d} \sigma_{c e} P_{ \pm} \odot \sigma^{e}{ }_{d} P_{\mp}=0
\end{aligned}
$$


Eq (68) is derived using eq (63):

$$
\begin{aligned}
\sigma^{\mu v} P_{ \pm} \odot \sigma_{\mu v} P_{\mp} & =\mp i \sigma^{\mu v} P_{ \pm} \odot \tilde{\sigma}_{\mu v} P_{\mp}=\mp i \frac{1}{2} \varepsilon_{\mu v \alpha \beta} \sigma^{\mu v} P_{ \pm} \odot \sigma^{\alpha \beta} P_{\mp} \\
& =\mp i \tilde{\sigma}_{\alpha \beta} P_{ \pm} \odot \sigma^{\alpha \beta} P_{\mp}=-\sigma^{\mu v} P_{ \pm} \odot \sigma_{\mu v} P_{\mp}=0
\end{aligned}
$$

Eq (69) is obtained by expressing both $\sigma$ 's in terms of $\tilde{\sigma}$ using eq (63), contracting the two $\varepsilon$ 's, and applying eq (68), while eq (70) is a result of symmetry in eq (69). Similar manipulations in the chirality-diagonal case yield

$$
\begin{aligned}
& \sigma^{a c} P_{\mp} \odot \sigma^{b}{ }_{c} P_{\mp}=\frac{1}{2} g^{a b} \sigma^{c d} P_{\mp} \odot \sigma_{c d} P_{\mp}-\sigma^{b c} P_{\mp} \odot \sigma^{a}{ }_{c} P_{\mp} \\
& \mp i \varepsilon^{a b c d} \sigma_{c e} P_{\mp} \odot \sigma^{e}{ }_{d} P_{\mp}=\sigma^{b}{ }_{d} P_{\mp} \odot \sigma^{a d} P_{\mp}-{ }^{a} \leftrightarrow^{b}
\end{aligned}
$$

\section{References}

[1] M. Fierz, Z. Phys. 104 (1937) 553.

[2] R. H. Good, Rev. Mod. Phys. 27 (1955) 187.

[3] J. F. Nieves and P. B. Pal, Am. J. Phys. 72 (2004) 1100 [hep-ph/0306087].

[4] C. C. Nishi, Am. J. Phys. 73 (2005) 1160 [hep-ph/0412245].

[5] L. Girlanda, Phys. Rev. C 77 (2008) 067001 [arXiv:0804.0772 [nucl-th]].

[6] S. -L. Zhu, C. M. Maekawa, B. R. Holstein, M. J. Ramsey-Musolf and U. van Kolck, Nucl. Phys. A 748 (2005) 435 [nucl-th/0407087].

[7] G. 't Hooft, Phys. Rept. 142 (1986) 357.

[8] See for instance: A. W. Steiner, Phys. Rev. D 72 (2005) 054024 [hep-ph/0506238]; H. Basler and M. Buballa, Phys. Rev. D 82 (2010) 094004 [arXiv:1007.5198 [hep-ph]].

[9] J. F. Kamenik and C. Smith, JHEP 1203 (2012) 090 [arXiv:1111.6402 [hep-ph]].

[10] Z. -H. Yu, J. -M. Zheng, X. -J. Bi, Z. Li, D. -X. Yao and H. -H. Zhang, Nucl. Phys. B 860 (2012) 115 [arXiv:1112.6052 [hep-ph]].

[11] R. Ding and Y. Liao, JHEP 1204 (2012) 054 [arXiv:1201.0506 [hep-ph]].

[12] W. Rarita and J. Schwinger, Phys. Rev. 60, 61 (1941). 